\title{
ADECUACIÓN DE LA NORMA ISO/IEC 29110 E IEEE 829 PARA LA GESTIÓN DE PROYECTOS DE DESARROLLO CON METODOLOGÍA ICONIX
}

\author{
Beatriz Eugenia Marín Ospina \\ Semillero ITMedia \\ Grupo Grintic \\ Institución Universitaria Antonio José Camacho \\ Recibido: 02/03/2020. Aprobado: 19/10/2020 \\ Cómo citar este artículo: \\ Marín Ospina, B.E. (2020). Adecuación de la norma ISO/IEC 29110 e IEEE 829 para la gestión de proyectos de \\ desarrollo con metodología Iconix. Revista Sapientía, 12(24), 6-15.
}

\section{RESUMEN}

Los productos de software deben garantizar el cumplimiento de criterios mínimos de calidad aplicando métodos y buenas prácticas propuestas a partir de las experiencias de autores y organizaciones que han trabajado en el tema por mucho tiempo. Estas experiencias brindan lineamientos que se pueden ajustar a diversos contextos. En este proyecto se presenta la adecuación de las prácticas propuestas por la norma ISO/IEC 29110 para gestión de proyectos de desarrollo en pequeñas empresas, integrando la metodología Iconix para guiar los procesos de desarrollo de software y la IEEE 829 para documentar las pruebas en el marco del desarrollo del módulo de transferencia y comunicación de datos de las estaciones ambientales de la empresa San Ambiente de Cali.

Este trabajo corresponde a la fase 1 del macroproyecto "Desarrollo de un aplicativo de gestión de datos ambientales para la empresa San Ambiente de Cali", perteneciente al semillero ITMedia de la Facultad de Ingenierías de la Institución Universitaria Antonio José Camacho.

\section{PALABRAS CLAVE}

ISO/IEC 29110, IEEE 829, Metodología Iconix, calidad de software.

\section{ABSTRACT}

Software products must ensure compliance with minimum quality criteria by applying methods and good practices that have been proposed based on the experiences of authors and organizations that have worked on the subject for a long time. These experiences provide guidelines that can be adjusted to different contexts.

This project presents the adaptation of the practices proposed by the ISO / IEC 29110 standard for management of small business development projects integrating the Iconix methodology to guide the software development processes and the IEEE 829 to document the tests in the framework of 
the development of the module of transfer and communication of data of the environmental stations of the company San Ambiente de Cali.

This work corresponds to phase 1 of the macroproject "Development of an application of environmental data management for the company San Ambiente de Cali" of the ITMedia research seedbed of the Faculty of Engineering of the Institución Universitaria Antonio José Camacho.

\section{KEYWORDS}

ISO/IEC 29110, IEEE 829, iconix methodology, software quality.

\section{INTRODUCCIÓN}

La industria del software en Colombia al año 2018 agrupaba 6.096 empresas de las cuales el 90\% correspondían a Pymes (Piedrahíta, 2018). Estas cifras traen consigo la necesidad de ser cada vez más competitivas si se quieren mantener vigentes en el mercado. Para ello las empresas se están esforzando por procurar desarrollos de calidad a través de la utilización de métodos de gestión que han demostrado su efectividad a nivel mundial. Estándares como el PMBOK agrupan buenas prácticas para guiar la administración de los proyectos y metodologías como Prince 2 plantean roles, responsabilidades y procesos necesarios para planear, diseñar y ejecutar un proyecto.

Para las Pymes la utilización de estos estándares y métodos exigen una inversión importante, ya que requieren personal altamente capacitado que pueda articularlos con la cultura organizacional (Legaria 2018). Por esta razón, la International Organization for Standardization (ISO) en alianza con la International Electrotechnical Commission (IEC) propuso la norma ISO/IEC 29110 que busca orientar la gestión de los proyectos en las pymes, buscando facilitar el control de sus procesos básicos y la disminución de riesgos. Esta norma puede verse como el primer nivel de madurez que da paso a otros modelos más avanzados que llevan a medir y mejorar los procesos. Cuenta, además, con procesos de implementación de software que guían al equipo de desarrollo, sin embargo, de acuerdo con el contexto particular de la empresa o el grupo de trabajo, estos procesos deben adaptarse a las particularidades de la organización.

La Facultad de Ingenierías de la Institución Universitaria Antonio José Camacho, con sus semilleros de investigación, en convenio con algunas empresas se encuentra desarrollando productos de software desde hace varios años. Esta dinámica ha requerido incluir estrategias de gestión para garantizar el éxito de los proyectos convirtiendo el espacio del semillero en una pequeña organización conformada por estudiantes, docentes y empresarios, donde se realizan productos de software a la medida facilitando el estudio y puesta en práctica de diferentes técnicas y métodos enriqueciendo el quehacer académico.

A continuación se presenta el marco resultante de la construcción de la primera fase del macroproyecto "Desarrollo de un aplicativo de gestión de datos ambientales para la empresa San Ambiente de Cali" del Semillero de Investigación en Tecnologías Multimedia (ITMedia), realizada a través de los proyectos de grado de Grajales y Pazú (2020), Lozano y otros (2020) y Romero y Posú (2020), que vincula a los procesos de gestión de la ISO 29110, los procesos de implementación de software que propone la metodología Iconix y las etapas de documentación de pruebas de la IEEE 829.

\section{MARCO TEÓRICO}

La norma ISO/IEC 29110 propone un conjunto de prácticas aplicables en pequeñas organizaciones (PO) para facilitar la gestión del ciclo de vida de un producto de software (Pino, Pino, y Delgado 2019); está conformada por 5 partes cada una orientada a un público objetivo. La primera parte presenta la visión general de los procedimientos, en la 
segunda parte están los conceptos de perfiles y su lógica, en la tercera parte se definen los esquemas de evaluación para certificación, en la cuarta parte está la especificación para todos los perfiles y por último en la parte 5 está la guía de administración e ingeniería enfocada en pequeñas empresas con un conjunto de reportes técnicos de acuerdo con los subgrupos dentro del grupo de perfiles.

El perfil genérico es la categoría más general y se refiere a empresas que no desarrollan sistemas o productos de software críticos bajo contextos típicos y contiene varios subgrupos, el de nivel más bajo es el perfil de entrada y está dirigido a PO con no más de tres años de fundación o con un número de empleados menor o igual a seis. El siguiente perfil es el básico y está orientado a $\mathrm{PO}$ que desarrollan una sola aplicación con un solo equipo. El perfil intermedio está dirigido a $\mathrm{PO}$ que desarrollan más de un proyecto con más de un equipo de trabajo. Por último se encuentra el perfil avanzado que se enfoca en empresas que desean ser competitivas y mantenerse en el mercado (ISO 29110 s/f). La norma propone también un conjunto de paquetes de despliegue que consisten en artefactos ya desarrollados que sirven de referencia para la implementación de los procesos. Para el perfil básico se tienen paquetes de despliegue para análisis de requisitos, arquitectura y diseño detallado, construcción y pruebas unitarias, integración y pruebas, verificación y validación, control de versiones, gestión del proyecto, entrega del producto y autoevaluación (Laporte, O'Connor, y García-Paucar 2016).

La norma propone también un conjunto de paquetes de despliegue que consisten en artefactos ya desarrollados que sirven de referencia para la implementación de los procesos. Para el perfil básico se tienen paquetes de despliegue para análisis de requisitos, arquitectura y diseño detallado, construcción y pruebas unitarias, integración y pruebas, verificación y validación, control de versiones, gestión del proyecto, entrega del producto y autoevaluación (Laporte, O’Connor, y García-Paucar 2016).
Los procesos de gestión y de implementación del software propuestos por la norma se muestran en la figura 1.

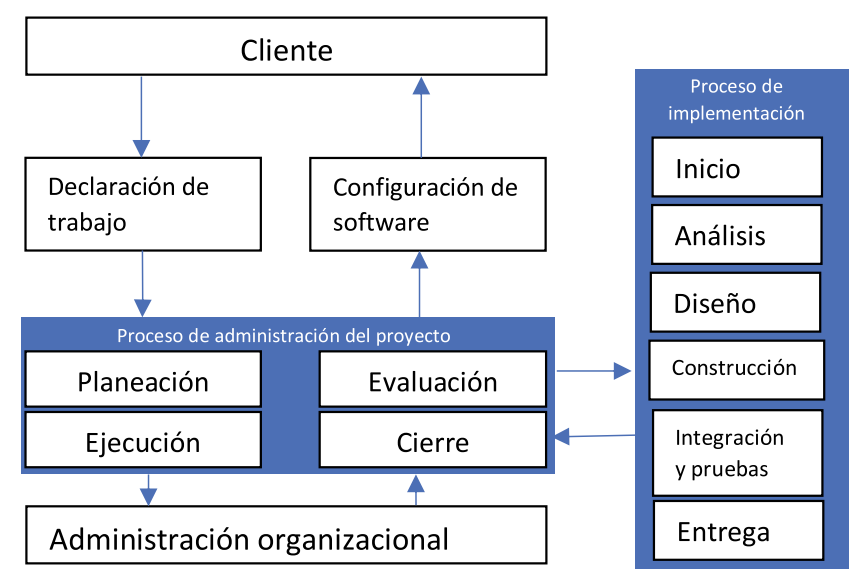

Figura 1. Perfil básico con sus procesos y actividades según la norma ISO/IEC 29110.

Fuente: adaptado de Laporte et al. (2016)

Bajo este marco se han desarrollado varias investigaciones que buscan demostrar su efectividad en diferentes contextos, uno de ellos corresponde al trabajo de Madruñero (2018) que pretendía dar formalidad a la gestión de los desarrollos en la Unidad de dirección de desarrollo de la Universidad Técnica del Norte. Las producciones de software en esta unidad se han enmarcado con elementos de Scrum, pero sin ninguna rigurosidad. Las etapas de este proyecto inician con el estudio documental del marco, el conocimiento del negocio y terminan con la propuesta de un método híbrido de integración de la norma con la metodología. Una conclusión para destacar es la dificultad de articular esta norma con las metodologías ágiles; el marco resultante combinó características, roles y funciones de Scrum con criterios de documentación de metodologías tradicionales siguiendo las fases de inicio, planificación, ejecución-evaluación y cierre.

En el trabajo de Laporte y otros (2016) se presenta la aplicación de esta norma en 7 casos distintos que comprenden pequeñas empresas del Perú y Canadá, demostrando su efectividad para planear y ejecutar proyectos sin interferir en la creatividad de los desarrolladores, teniendo en 
cuenta indicadores como costos y tiempo. De igual manera, en el estudio de Legaria (2018) se plantea la implementación de la norma para la gestión de proyectos en una pequeña empresa de infraestructura tecnológica adecuando los subprocesos de planeación, ejecución, evaluación y cierre a las actividades técnicas de infraestructura, mostrando la flexibilidad de la norma para adaptarse a otro tipo de proyectos.

Finalmente, en el trabajo de Castiblanco (2019) se propone un modelo de gestión basado en la norma ISO/IEC 29110, tomando como referencia una empresa pequeña de la ciudad de IbaguéColombia. El autor compara diversos métodos de gestión y de desarrollo de software presentando algunos híbridos que han surgido como Q-Scrum y Pmbok con la ISO 29110, entre otros.

\section{METODOLOGÍA}

Los pasos para el desarrollo del proyecto fueron los siguientes:

\section{Caracterización del contexto}

El proceso parte de la identificación del equipo de trabajo en el semillero ITMedia, presentado en la tabla 1, donde se analizaron las debilidades $y$ fortalezas que permitieron identificar los componentes que se necesitaban en el proceso de gestión y desarrollo del software.

Tabla 1. Variables que describen los equipos de trabajo en el semillero ITMedia de la UNIAJC.

\begin{tabular}{ll}
\hline Variable & Descripción \\
\hline $\begin{array}{l}\text { Tamaño de los equipos de } \\
\text { trabajo }\end{array}$ & $\begin{array}{l}\text { Entre 1 y } 3 \text { estudiantes en } \\
\text { cada grupo. Se tienen } 4 \\
\text { grupos. }\end{array}$
\end{tabular}

\begin{tabular}{ll}
\hline Duración de los proyectos & Entre 12 y 18 meses \\
\hline Experiencia en el área & $\begin{array}{l}\text { En su mayoría solo trabajos } \\
\text { académicos }\end{array}$
\end{tabular}

Restricciones:

- La dedicación horaria de los integrantes del semillero es parcial.

- Las reuniones de integración y validación con el usuario final son limitadas a encuentros mensuales.

- Las reuniones grupales donde se encuentran todos los equipos que conforman el macroproyecto tienen una frecuencia mensual.

- Se deben cumplir los lineamientos mínimos del programa académico de Ingeniería de Sistemas para la realización de proyectos de grado.

Las características del equipo y la dinámica de trabajo implicaban mantener documentación suficiente que evidenciara los procesos de análisis, diseño, implementación y pruebas facilitando la incorporación de estudiantes en cualquier etapa, pero sin llegar al extremo de sobrecarga de documentos. Además, era necesario cumplir con requisitos de calidad rigurosos que le garantizaran a la empresa que recibe el producto un nivel aceptable de funcionalidad, mantenibilidad y usabilidad.

\section{Revisión de normas y métodos}

A partir de las características y restricciones anteriores se planteó la utilización de los procesos de gestión de la ISO/IEC 29110 y una metodología de desarrollo de software que combinara la agilidad en la producción con la documentación mínima necesaria para permitir la trazabilidad de los procesos, encontrando que Iconix combinaba la metodología RUP con XP, utilizando como base de la comunicación los casos de uso y el prototipado (Silva \& García, 2018) resolviendo el problema de las distantes reuniones con el usuario y disminuyendo el tiempo de entrenamiento de los nuevos semilleristas en el proyecto. Además en otros estudios se ha demostrado superioridad de la calidad del producto producido con Iconix frente a otras metodologías (Porras 2010). En la figura 2. se presentan los procesos de Iconix. 


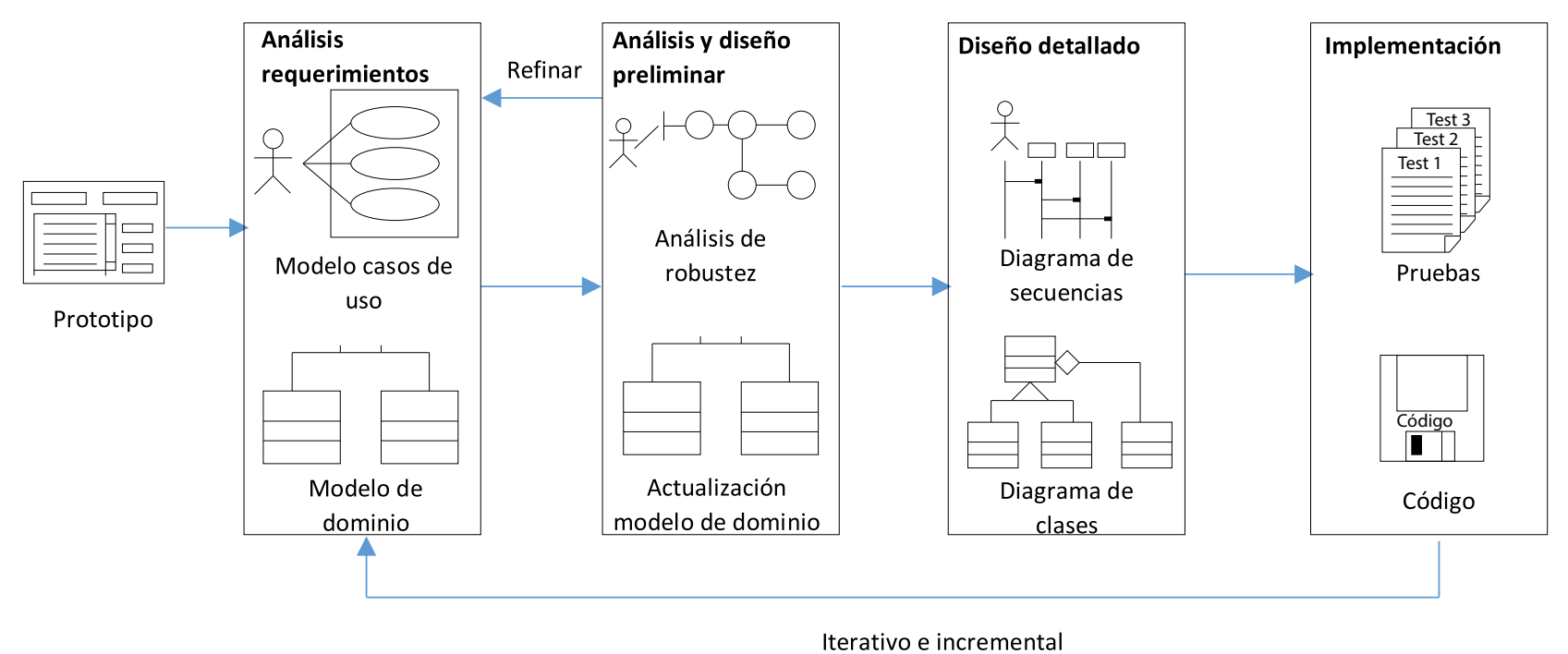

Figura 2. Descripción del proceso de Iconix.

Fuente: elaboración propia.

Posteriormente se compararon las características de la ISO 29110 con la metodología ICONIX para validar sus semejanzas y compatibilidad (Tabla 2).

Tabla 2. Comparación de la norma ISO 29110 con la metodología Iconix.

\begin{tabular}{llll} 
Características & ISO $29110-$ Gestión del proyecto & ISO 29110-Proceso de & Iconix \\
\hline Roles & implementación del software & \\
& 1.Cliente & 1.Cliente & 1.Jefe de proyecto \\
& 2.Gestor de proyecto & 2.Analista & 2.Analista de sistemas \\
3.Líder técnico & 3.Diseñador & 3.Programador \\
4.equipo de trabajo & 4.Programador & 4.Ingeniero de software \\
& & 5.Gestor del proyecto & \\
& & 6.Líder técnico & \\
\hline Actividades & 7.Equipo de trabajo & 1. Análisis de requisitos \\
& 1. Planificación del proyecto & 1. Inicio de implementación de & 2. Análisis y diseño preliminar \\
& 2. Ejecución del plan del proyecto & software & 3. Diseño \\
& 3. Evaluación y control del & 2. Análisis de requisitos & 4. Arquitectura y diseño detallado \\
proyecto & 4el software & \\
4. Cierre del proyecto & 4. Construcción del software & \\
& 5. Integración y pruebas del software \\
& 6. Entrega del producto & \\
\hline
\end{tabular}


Viene de la pág. 10

\begin{tabular}{llll}
\hline Productos & Externos: & 1.Especificación de requisitos & 1.Modelo de dominio \\
1.Plan del proyecto & 2.Diseño de software & 2.Modelo de casos de uso \\
2.Declaración de trabajo & 3.Registro de trazabilidad & 3.Prototipo de interfaz \\
3.Repositorio del proyecto & 4.Componente de software & 4.Diagrama de robustez \\
4. Acta de reunión & 5.Software & 5.Codificación \\
5. Configuración del software & $\begin{array}{l}\text { 6.Casos de prueba y procedimientos } \\
\text { de pruebas }\end{array}$ & 6.Pruebas \\
Internos: & 7.Reporte de pruebas \\
6.Solicitud de cambio & 8.Manual de operación & \\
7.Acciones correctivas & 9.Manual de usuario & \\
8.Resultados de verificación & 10.Manual de mantenimiento & \\
9.Registro de estado del proyecto & & \\
10.Respaldo del repositorio & & \\
\end{tabular}

Fuente: elaboración propia.

Lo que se puede observar en la tabla 2, es que los roles son similares tanto en la ISO como en Iconix. El número de roles es menor en Iconix. En cuanto a los procesos son similares para el desarrollo de software. La cantidad de productos de desarrollo de software también son menores en Iconix.

Tanto en la metodología Iconix como en la norma ISO se identifica el proceso de pruebas como un requisito de aseguramiento de la calidad. Los métodos de diseño e implementación se encuentran detallados en el estándar IEEE 829 que en proyectos anteriores han dado muy buen resultado (Aguado 2019; Romero y Posú 2020), por esta razón se incorpora este estándar para el proceso de pruebas. En la figura 3 se presentan los productos y las etapas que propone.

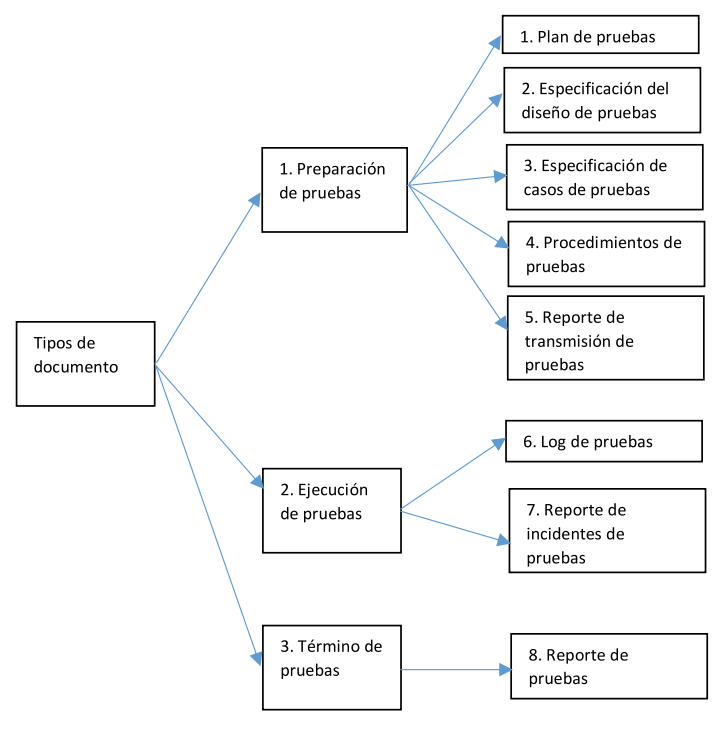

Figura 3. Plan de documentación de pruebas del estándar IEEE 829.

Fuente: tomado de Romero y Posú (2020)

\section{Ejecución del proceso de gestión y desarrollo del producto}

Después de identificar las normas y métodos dentro de un único marco se procedió a iniciar el proyecto de transferencia y comunicación de datos para la empresa San Ambiente con el fin de determinar la pertinencia de los productos en cada etapa.

\section{Refinamiento del marco propuesto}

Con base en las lecciones aprendidas durante el desarrollo se refinaron los procedimientos y artefactos utilizados.

\section{RESULTADOS}

El proceso de gestión del proyecto siguió las directrices de la ISO/IEC 29110 integrando en un 
solo documento los resultados de la verificaciónregistro de avance del proyecto $\mathrm{y}$ en otro documento el registro de corrección-solicitud de cambio, quedando como se muestra en la figura 4 .

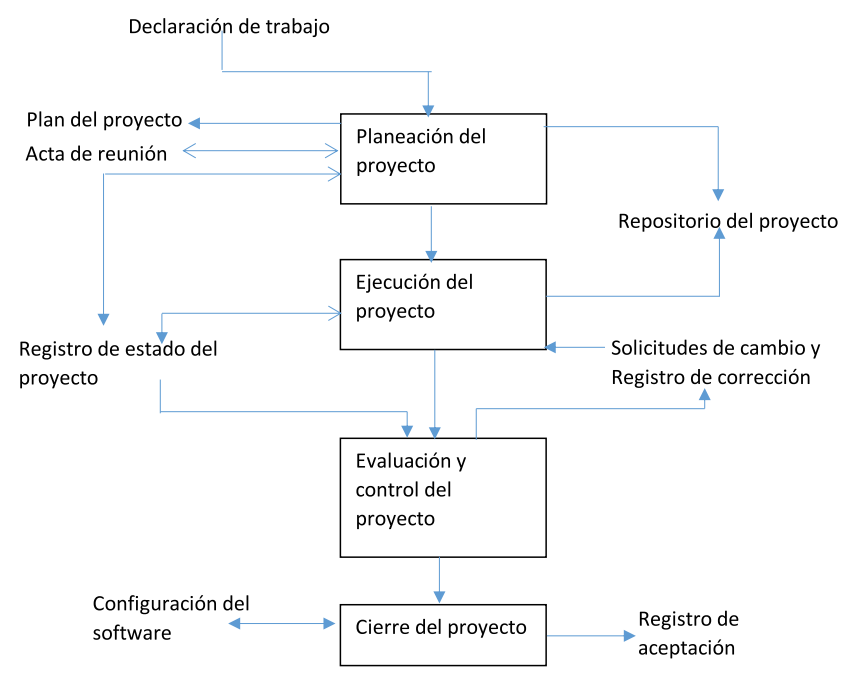

Figura 4. Proceso adecuado de gestión y documentos a partir de la norma ISO/IEC 29110.

Fuente: elaboración propia.

Cada una de las etapas se presenta a continuación:

- En la etapa de planificación se especificó el documento del plan de proyecto con la descripción del producto, propósito general del negocio, requisitos generales del cliente, objetivos del producto, entregables al usuario, tareas y duración estimada; recursos humanos y técnicos necesarios, composición del equipo de trabajo (1 equipo de calidad conformado por dos estudiantes, 1 equipo de desarrollo conformado por 3 estudiantes y el equipo de gestión conformado por 2 estudiantes más); calendario de las tareas del proyecto, costos estimados, estrategia de control de versiones (se utilizó GitHub y los equipos personales como respaldo adicional). Otros productos generados en esta etapa son el acta de reunión donde se revisó y validó el plan de proyecto y el repositorio montado en la nube. El documento de resultados de verificación fue incluido dentro del análisis en la declaración del trabajo.

- En la etapa de ejecución del plan de proyecto se inició la implementación de la metodología Iconix con la IEEE 829, actualizando en cada una de las subfases el repositorio, el registro de estado del proyecto generando las solicitudes de cambio y registros de corrección correspondientes. Para la primera iteración fue necesario actualizar el calendario de las tareas del proyecto existente en el plan de proyecto debido al aumento de tiempo en la curva de aprendizaje. El documento de solicitudes de cambio y registro de corrección fueron unificados en un solo artefacto.

- En la etapa de evaluación y control del proyecto se realizó por cada grupo de casos de uso un diagrama comparativo del tiempo propuesto para su desarrollo en cada una de sus fases y el tiempo real utilizado, con el fin de generar indicadores que pudieran servir de referencia para futuros proyectos y actualizar fácilmente el registro de estado del proyecto. En esta fase también se generaron solicitudes de cambio y registro de corrección.

- En la etapa de cierre del proyecto se entrega el código fuente, manuales e instaladores al cliente y se firma un registro de aceptación del producto. En este momento se han cerrado los módulos de parametrización y, a final de marzo del presente año, se cierra el módulo de comunicación.

El proceso de desarrollo del software siguiendo la metodología Iconix y la IEEE 829 se muestra en la figura 5. 


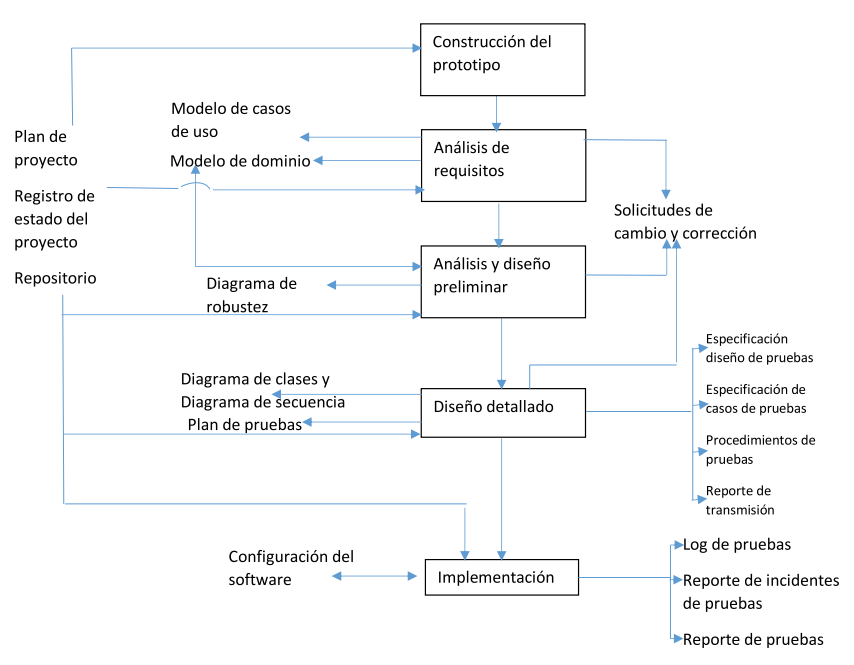

Figura 5. Metodología y productos de Iconix con el estándar IEEE 829 guiada por la norma ISO/IEC 29110.

Fuente: elaboración propia.

La metodología de desarrollo del software Iconix toma comoinsumo el plan de proyecto del proceso de gestión, donde se describieron tareas y calendarios generales. A partir de los requerimientos generales se construyó un prototipo base por parte del equipo de desarrollo y a partir de este producto se ejecutaron las siguientes fases:

- Enla fase deanálisis de requisitos se desarrollaron las clases conceptuales del problema y el modelo de casos de uso. Se registraron los cambios en las solicitudes de cambio y corrección, el repositorio y el registro del estado del proyecto.

- En el análisis y diseño preliminar se refinó el modelado del dominio, se crearon los diagramas de robustez para presentar lo que se quiere hacer y asociarlo con la forma de hacerlo, actualizando el repositorio, solicitudes de cambio y corrección y el registro del estado del proyecto.

- En la etapa de diseño detallado se realizaron los diagramas de clases a partir de los diagramas de robustez y los diagramas de secuencias. También se diseñaron los documentos de plan de pruebas, especificación del diseño de pruebas, especificación de los casos de pruebas, los procedimientos de pruebas y los reportes de transmisión propuestos por la IEEE 829.

- Los atributos que se querían probar se identificaron a partir de la norma ISO/IEC 25010 en acuerdo con el usuario y se listan a continuación: adecuación funcional, eficiencia, usabilidad y seguridad. Las herramientas para realizar las pruebas fueron escogidas por comparación y estudio documental utilizando finalmente Jest, JMeter, Visual Studio Code, Angular, Postgresql, entrevistas para usabilidad y revisión de la funcionalidad a partir de los casos de uso (Romero y Posú 2020). Se actualiza el registro de estado del proyecto, el repositorio y se diligencian las solicitudes de cambio y corrección necesarios.

- En la etapa de implementación se desarrollaron los productos de software acordados en el plan del proyecto, generando logs de pruebas, reportes de incidentes y reporte general de pruebas, actualizando el registro del estado del proyecto y el repositorio.

\section{CONCLUSIONES}

$\mathrm{Al}$ analizar los procedimientos de la ISO el equipo se ubicó en el perfil básico por el número de integrantes, tiempo y viabilidad del proyecto. Los artefactos del paquete de despliegue contenían la información necesaria para facilitar la trazabilidad del desarrollo, pero en algunos casos se encontró redundancia en el registro de la información por esta razón se unificaron las solicitudes de cambios y los registros de corrección.

El uso de Iconix permitió disminuir el número de documentos en la configuración del software haciendo ágil el proceso. Además, la elaboración de prototipos en las etapas tempranas del ciclo vida facilitó la comunicación con el usuario final.

La integración de los pasos y documentos establecidos en la IEEE 829 dentro de las etapas de diseño detallado e implementación de Iconix 
condujo a procedimientos bien estructurados con resultados positivos en la calidad final del producto.

\section{RECOMENDACIONES Y TRABAJOS FUTUROS}

En este proyecto se buscó aplicar técnicas que facilitaran la organización, trazabilidad y gestión de los procesos en torno a las características del equipo de trabajo, para cumplir con los requisitos del programa académico y garantizar un producto de calidad para la empresa. Inicialmente se realizaron varias iteraciones donde se fueron refinando las etapas y productos propuestos por las normas y la metodología. El resultado final fue un producto desarrollado bajo un marco de trabajado académico, pero cumpliendo estándares utilizados en la industria.

Se obtuvieron muchas lecciones aprendidas que permitieron ajustar los lineamientos de las normas y métodos a las necesidades del grupo. Estas lecciones han sido la mayor ganancia para el equipo, permitiendo confrontar las dificultades que se pueden llegar a tener en la industria.

El trabajo siguiente consiste en desarrollar la fase 2 del proyecto de San Ambiente donde se procesan los datos cargados desde las estaciones y que permitirá validar y refinar los métodos propuestos, bajo un nuevo contexto definido por las características propias de los nuevos miembros del semillero.

\section{REFERENCIAS BIBLIOGRÁFICAS}

Aguado, J. (2019). Implementación de pruebas de calidad en la aplicación control currículo v.1 de la Institución Universitaria Antonio José Camacho (Tesis de Pregrado). Institución Universitaria Antonio José Camacho, Cali, Valle, Colombia.

Castiblanco, D. (2019). Diseño del Proceso Gestión de Proyectos GP del Modelo de Calidad ISO/IEC 291104-1 Perfil Básico para la Empresa TIC Makers S.A.S.
(Tesis Especialización en Gestión de Proyectos). Universidad Nacional Abierta y a Distancia UNAD, Cali, Valle, Colombia.

Grajales, A. y Pazú, C. (2020). Desarrollo de un marco de trabajo siguiendo el estándar ISO/IEC 29110 para el caso de estudio de integración de procesos del proyecto San Ambiente (Tesis de Pregrado Ingeniería de Sistemas). Institución Universitaria Antonio José Camacho, Cali, Valle, Colombia.

ISO. (s.f.). ISO/IEC TR 29110-1:2016(es), Ingeniería de Software y Sistemas - Perfiles de ciclo de vida para Pequeñas Organizaciones (VSEs) Parte 1: Visión general. Recuperado el 11 de junio de 2020 https://www.iso.org/obp/ui\#iso:std:isoiec:tr:29110:-1:ed-2:v1:es

Laporte, C., O’Connor, R. y García-Paucar, L.H. (2016). The implementation of ISO/IEC 29110 software engineering standards and guides in very small entities. In: Maciaszek, L.A. and Filipe, Joaquim, (eds.) Evaluation of Novel Approaches to Software Engineering. CCIS, 599. Springer International Publishing, pp. 162-179.

Legaria, D. (2018). Implementación de procesos organizacional de gestión de proyectos en Developit (Tesis de Maestría). Universidad de Chile, Santiago de Chile.

Lozano, J., Bolivar, J. y Ramírez, J.J. (2020). Desarrollo de los módulos de transferencia y comunicación de datos ambientales para la empresa de SANAMBIENTE de Cali (Tesis de Tecnología en Sistemas de Información). Institución Universitaria Antonio José Camacho, Cali, Valle, Colombia.

Madruñero, E. (2018). Implementación del estándar ISO/ IEC 29110 en el proceso de desarrollo de software de la dirección de desarrollo tecnológico e informático de la Universidad Técnica del Norte (Tesis de Maestría). Universidad Técnica del Norte, Ecuador.

Piedrahíta, E. (2018). La clave es el 'software'. Revista Semana. 
Pino, F., Pino, A. y Delgado, B. (2019). ISO/IEC 29110, apoyo a las pequeñas organizaciones desarrolladoras de software. Recuperado el 1 de marzo de 2020 https://revista.aenor.com/344/isoiec-29110-apoyo-alas-pequenas-organizaciones-desarrollad.html

Porras, E. (2010). Comparación de dos procesos de desarrollo de software usando los métodos ICONIX y $X P$, caso: Comercialización de la Tara en la región de Ayacucho (Tesis de Maestría). Universidad Nacional de Ingeniería, Lima-Perú.

Romero, D., y Posú, F. (2020). Aplicación de un modelo de calidad al módulo de comunicación y traspaso de información del sistema de gestión de datos ambientales de la empresa SANAMBIENTE (Tesis de Pregrado Ingeniería de Sistemas). Institución Universitaria Antonio José Camacho, Cali, Valle, Colombia.

Silva, S., y García, C. (2018). Documentación de la metolodogía iconix a través del desarrollo del caso Oriéntate Cali (Tesis de Pregrado Ingeniería de Sistemas). Institución Universitaria Antonio José Camacho, Cali, Valle, Colombia.

Vallecilla Benalcázar, R. (2019). Sistema Web de Inventario de bienes patrimoniales (Tesis de Pregrado). Universidad Central de Ecuador, Quito.

\section{AUTORA}

\section{Beatriz Eugenia Marín}

Ingeniera de Sistemas de la Universidad del Valle. Candidata a Master of Science in Geographical Information Systems de la Universidad de Salzburg Austria. Docente de la Institución Universitaria Antonio José Camacho de Cali, Colombia. Integrante del grupo de investigación Grintic de la misma institución. 